\title{
Magnetic control of structural changes in structural steels during plastic deformation
}

\author{
Valeriy Gordienko ${ }^{1, *}$ \\ ${ }^{1}$ State University of Architecture and Civil Engineering, 190005, St. Petersburg, Russia
}

\begin{abstract}
The analysis and consideration of the influence of the main factors on the damage of welded metal structures during long-term operation. The relationship between the strength of the magnetic field of the Hp scattering and structural changes in structural steels that occur during cold plastic deformation to different degrees is established. It is shown that at small degrees of deformation, the change in the magnetic parameter Hp is large. As they increase, the HP value decreases and tends to the values of the Earth's magnetic field. The data of microstructural analysis of the metal of structural steels are in good agreement with the results of the passive ferrosonde method of control. A method for evaluating the effect of cold plastic deformation on the magnetic properties of metal in structural steels has been developed.
\end{abstract}

\section{Introduction}

The technical diagnosis of welded metal structures (MS) confirms that the most common cause of damage and, as a result, failure is the appearance of fatigue cracks in welded joints with structural heterogeneity (the presence of a cast structure in the weld, coarse-grained and fine-grained in the heat-affected zone). A negative impact on the performance of elements of welded metal structures is also exerted by local zones of stress concentration (SC) that arise during long-term operation, where plastic deformation is observed to one degree or another. Some unfavorable factors that contribute to the initiation and development of cracks and, as a result, lead to failures and serious accidents, are undoubtedly one of the main reasons for the decrease in the reliability and safety of operation of welded metal structures in general [1-5].

Considering the above, conducting studies on assessing the degree of influence of the structural state of welded joint zones on the strength characteristics of metal structures, as well as on finding simple and reliable methods of metal hardening in the identified hazardous zones by forming microstructures with a given degree of dispersion with higher mechanical characteristics, seems very relevant.

One of the practical methods for the formation of a microstructure with a given grain size should include recrystallization annealing carried out after preliminary cold plastic deformation of the metal. By changing the degree of deformation and the temperature of the subsequent recrystallization annealing, one can obtain a given grain size [6]. Thus, the

* Corresponding author: vgordienko@,lan.spbgasu.ru 
authors of [7], using vacuum melting iron, showed that one of the necessary conditions for obtaining a very fine-grained structure in high-purity materials is multiple deformation at low temperatures, which contributes to the formation of a large number of recrystallization centers, as well as limiting their growth rate.

It was of considerable scientific and practical interest to conduct in-depth studies not on model alloys, but on typical representatives of various classes of structural steels, while assessing the effect of the degree of cold plastic deformation on the forming microstructure. For research, low-carbon steel 08ps was selected and low-alloyed steel 09G2S and 10KHSND, which were widely used in construction, engineering and other industries [8].

\section{Materials and methods}

The research methodology provided for control of the process of structural changes in the selected structural steels 08ps, 09G2S and 10KHSND using the passive flux-gate method and the method of metallographic analysis in the process of deformation and thermal exposure.

The passive flux-gate method is based on the relationship between the structure, magnetic and mechanical properties of ferromagnetic metals and alloys based on them. According to the developed methods, the method can be used to control structural changes in the metal that occur during plastic deformation and heat treatment, to identify in the welded joints and elements of welded metal parts dangerous local zones of stress concentration $(\mathrm{KH})$, to assess the degree of their danger and indirectly determine the effective stresses in them , that is, control of the stress-strain state (VAT) of the metal [9$16]$.

The advantages of the method include such features as [17-19]:

- lack of need for special preliminary preparation of the controlled surface, removal of a non-magnetic protective coating, if this coating does not exceed a thickness of $2 \mathrm{~mm}$;

- the possibility of magnetic control of closed loops and hard-to-reach spots of elements of welded MK;

- the possibility of application for permanent or periodic magnetic monitoring in the identified local zones of the SC and zones of welded joints.

\section{Results and discussion}

In microstructural analysis, a complex of various devices was used, including grindingpreparation plants and metallographic devices. The grinding equipment included: a Brilliant 220 precision cutting unit, an Opal 460 specimen press fitting unit, and a Saphir 560 grinding and polishing unit. Metallographic studies were performed on Carl Zeiss Axio Observer and DMI 5000 inverted metallographic microscopes with mechanized tables equipped with quantitative analysis systems Thixomet images.

The chemical analysis of the investigated structural steels is presented in table. 1.

Table 1. The chemical analysis of the investigated structural steels.

\begin{tabular}{|c|c|c|c|c|c|c|c|c|c|}
\hline \multirow{2}{*}{$\begin{array}{c}\text { Steel } \\
\text { grade }\end{array}$} & \multicolumn{9}{|c|}{ Chemical composition, \% } \\
\cline { 2 - 10 } & $\mathrm{C}$ & $\mathrm{Mn}$ & $\mathrm{Si}$ & $\mathrm{Al}$ & $\mathrm{P}$ & $\mathrm{S}$ & $\mathrm{Cr}$ & $\mathrm{Ni}$ & $\mathrm{Cu}$ \\
\hline 08PS & 0,06 & 0,27 & 0,05 & 0,0011 & 0,019 & 0,022 & - & - & - \\
\hline 09G2S & 0,10 & 1,36 & 0,50 & - & 0,029 & 0,031 & - & - & - \\
\hline 10HSND & 0,12 & 0,57 & 0,92 & - & 0,032 & 0,036 & 0,63 & 0,87 & 0,48 \\
\hline
\end{tabular}

Samples for studies from mild steel 08ps and low alloy steels 09G2S and 10HSND in the state of factory supply were subjected to cold plastic deformation (rolling) to the degree 
$\varepsilon=8,15,22,30,40$, and $50 \%$. The scattering magnetic field Hp was measured with an IKNM-2FP device on the surface of the samples at three points spaced $30 \mathrm{~mm}$ apart from each other in the central part. In figures $1 \mathrm{a}-1 \mathrm{c}$ show the dependences of the magnetic parameter $\mathrm{Hp}$ on the degree of cold plastic deformation $\varepsilon$ at three points in the central part of the samples from low-carbon steel 08ps and low-alloyed 09G2S and 10HSND.

It can be seen (Fig. 1a) that with 08ps steel, with an increase in the degree of deformation at points 1 and 2, the magnetic parameter $\mathrm{Hp}$ changes negative values to positive ones, and after deformation $\varepsilon=30 \%$ it again changes sign. At the same time, the Hp value at the third point, regardless of the degree of cold plastic deformation, is in the negative region. However, at $\varepsilon=30 \%$, the magnitudes of the scattering magnetic field $\mathrm{Hp}$ at all points approach each other to their minimum values.

The initial Hp values for low-alloy steel 09G2S have different meanings: both negative and positive. With an increase in the degree of plastic deformation $\varepsilon$ of more than $22 \%$, the $\mathrm{Hp}$ values at all controlled points acquire only negative values. However, at the same time, the magnetic parameter $\mathrm{Hp}$ at the central point 2, like 08ps steel, lies between the values of neighboring points 1 and 3 .

At the same time, in 10HSND steel, all the initial values of the magnetic parameter Hp, in contrast to $08 \mathrm{PS}$ and $09 \mathrm{G} 2 \mathrm{~S}$, are only in the positive region. After deformation by $\varepsilon=$ $8 \%$, they decrease and take negative values. It should be emphasized that for all the studied steels with an increase in $\varepsilon$ of more than $15 \%$, the Hp values take a negative sign.

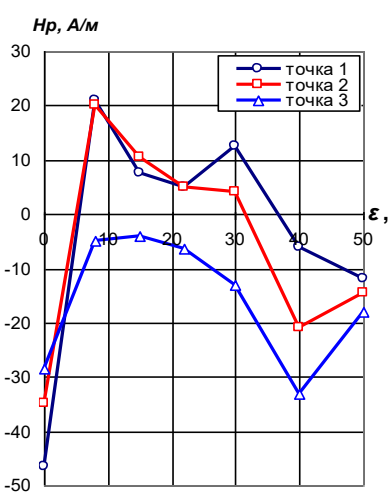

a)

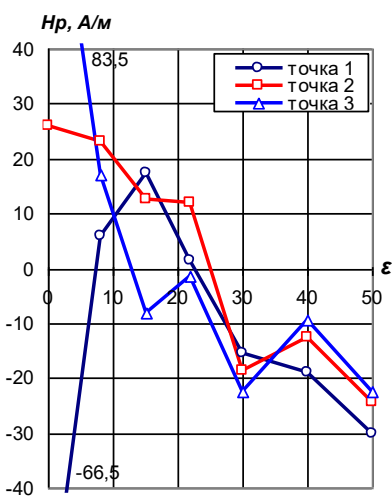

b)

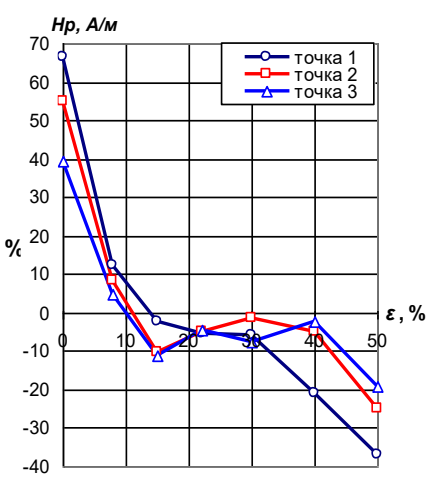

c)

Fig. 1. Dependence of the magnetic parameter $\mathrm{Hp}$ on the degree of plastic deformation $\varepsilon$ for steel a) 08PS, b) 09G2S, c) 10HSND.

In Figure 2 shows the dependence of the average values of the scattering magnetic field $\mathrm{Hp}$ on the degree of cold plastic deformation $\varepsilon$ for 08PS, 09G2S and 10HSND steels. 


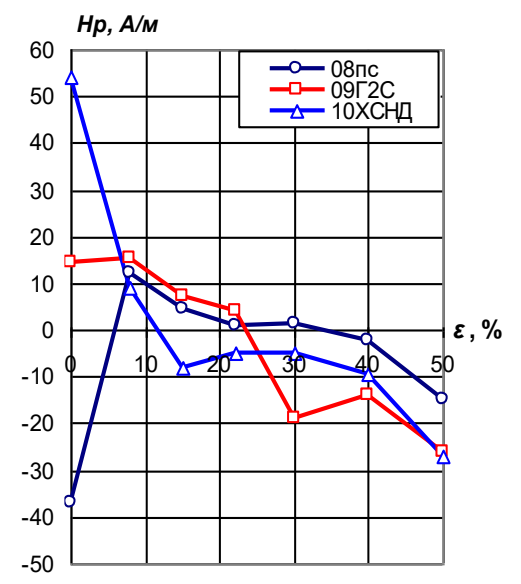

Fig. 2. Dependence of the magnetic parameter $\mathrm{Hp}$ on the degree of cold plastic deformation $\varepsilon$ for 08PS, 09G2S, and 10HSND steels.

It is seen that in the initial state there is a large scatter in the values of the Hp parameter and its different signs, however, with an increase in the degree of cold plastic deformation, the existing scatter decreases, and already at $\varepsilon=40 \%$ or more, all values of the Hp parameter acquire a negative sign. At $\varepsilon=50 \%$, the values of the Hp parameter for low alloy steels 09G2S and 10HSND become almost equal. Large changes in HP from the degree of deformation $\varepsilon$ in structural steels in magnitude and sign are largely due to structural changes in steels. The microstructure of the studied steels is shown in Fig. 3-5.

The microstructure of $08 \mathrm{ps}$ mild steel after plastic deformation to different degrees of $\varepsilon$ is shown in Fig. 3. It is seen that with an increase in the degree of deformation, an increase in the crystallographic orientation of grains occurs, however, the texture formation at small stages of rolling is weakly expressed; therefore, even after deformation by $\varepsilon=50 \%$, slightly deformed grains are visible in its structure, which confirms the results of a slight change in the parameter Hp even after fractional rolling by $\varepsilon=50 \%$.

In the structure of low-alloy steel 09G2S (Fig. 4), with an increase in the degree of plastic deformation, an increase in the crystallographic orientation of grains along the rolling is observed. But, nevertheless, at the initial stages of deformation, unreformed grains are visible, at the same time, there are grains with a significant degree of orientation near them. It should be noted that even at $\varepsilon=30 \%$, grains that would not undergo plastic deformation are not observed in the steel structure. 


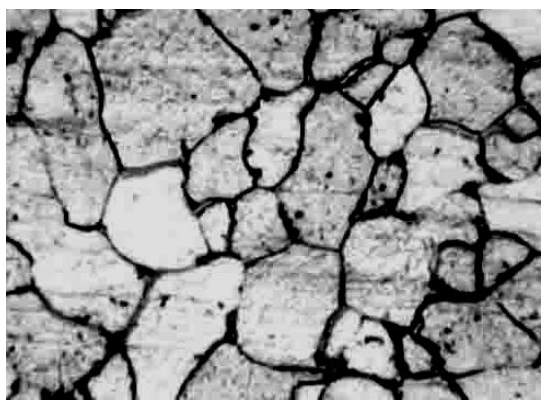

a)

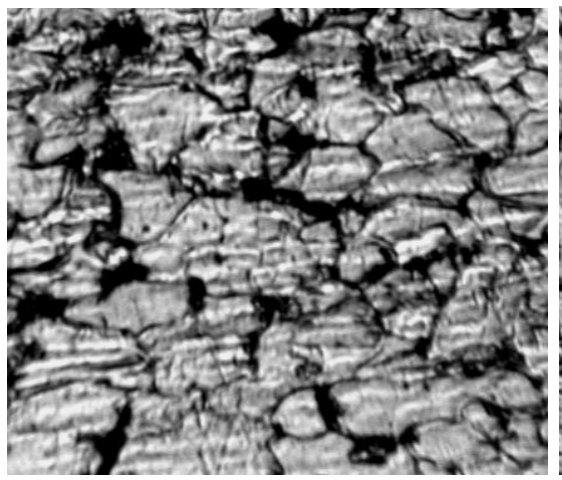

c)

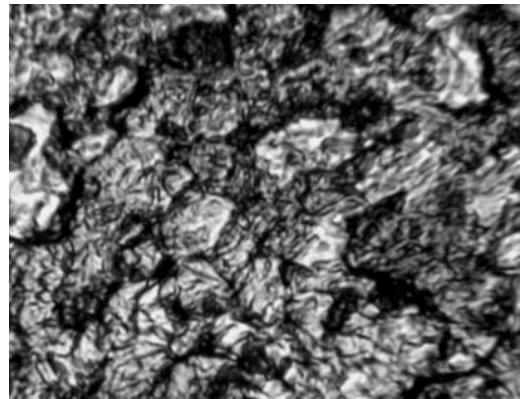

b)

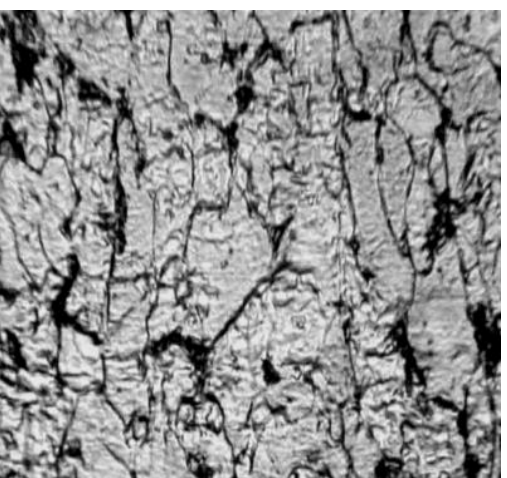

d)

Fig. 3. Microstructure of steel 08PS, X650: a) - state of factory supply, b) - d) - after deformation by the degree $\varepsilon=15,30,50 \%$.

Let us consider in more detail the structural changes in the fine structure of a metal that occur during plastic deformation. It was shown in [20] that if dislocations are distributed randomly in technical iron prior to plastic deformation, then after it is carried out, a dislocation forest (with a relatively uniform distribution of a large number of dislocations) and a coil structure, whose volume is approximately equal, are formed at $\varepsilon=10 \%$. During the formation of a glomerular dislocation structure, a redistribution of the dislocation density and the interaction of dislocations occurs with the formation of a structure that is more stable than the former. In our case, the ordering of the dislocation structure probably leads to a convergence of the magnetic parameter $\mathrm{Hp}$ in the steels under study at a strain of $\varepsilon=8 \%$ (Fig. 2). 


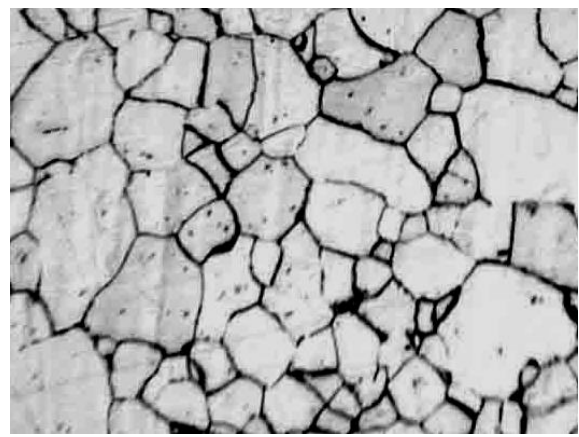

a)

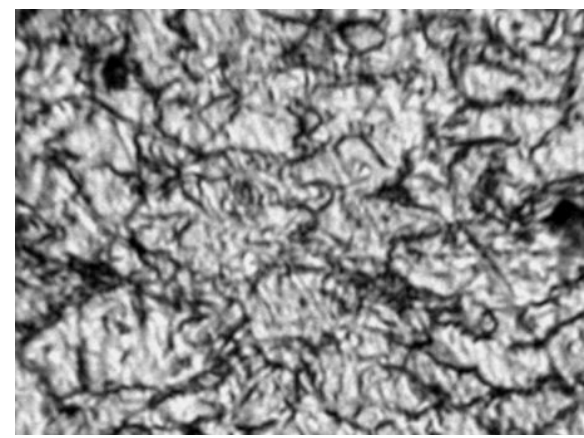

c)

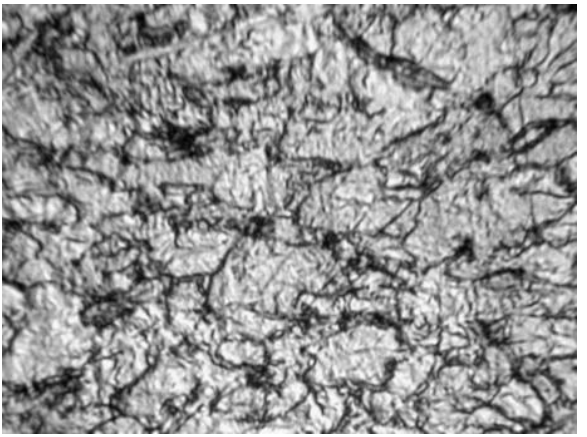

b)

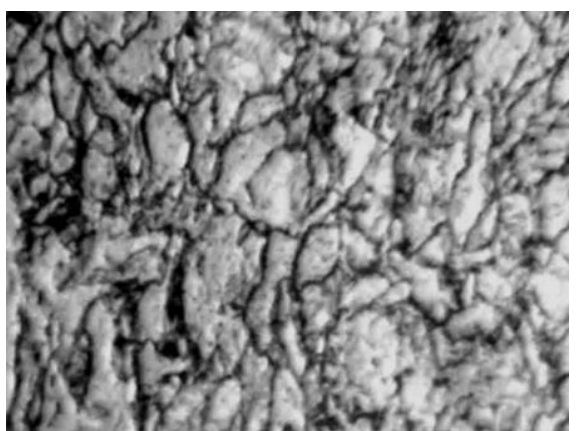

d)

Fig. 4. Microstructure of steel 09G2S, X650: a) - state of factory supply, b) - d) - after deformation to the degree $\varepsilon=15,30,50 \%$.

An increase in the degree of deformation to $\varepsilon=25 \%$ leads to the formation of a cellular structure, which is characterized by wide boundaries with a higher density of dislocations and relatively free from dislocations inter-boundary volumes of metal. When the steel is deformed to a degree of $\varepsilon=40 \%$, a strip structure is formed having elongated volumes that are bounded by fairly narrow walls. Its feature is the presence of strong disorientation, which is introduced by extended walls.

The change in the positive sign of $\mathrm{Hp}$ at a degree of deformation above $\varepsilon=22 \%$ by a negative one and subsequent changes in the values of $\mathrm{Hp}$ are apparently associated with the formation of cellular and then strip dislocation structures.

The authors of [21] on steel 10 during electron microscopic studies noted that with an increase in the degree of plastic deformation, the formation of plexus and thickening of dislocations, as well as the appearance of zones with a cellular dislocation structure, the density of dislocations reaching $8 \quad 108 \ldots 1,2109 \mathrm{~cm}^{-2}$. The most intense formation of the cellular structure occurred in perlite colonies. The authors believe that the presence of defects in the metal structure in the form of clusters of dislocations leads to stabilization of the domain walls in a weak magnetic field of the Earth, and they, due to the presence of long-range stress fields, form "long-range" domain walls that are able to form intrinsic scattering magnetic fields on the surface of the studied samples in the available stress concentration zones. 


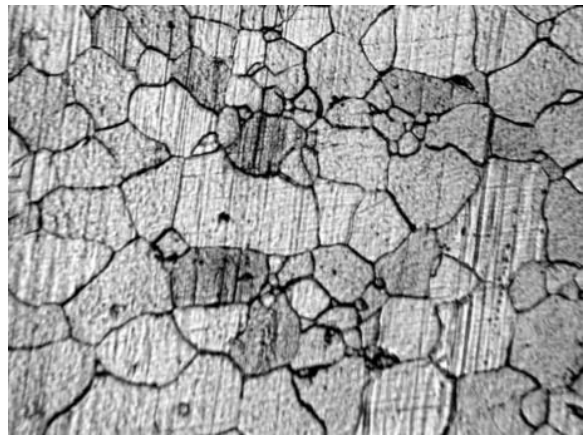

a)

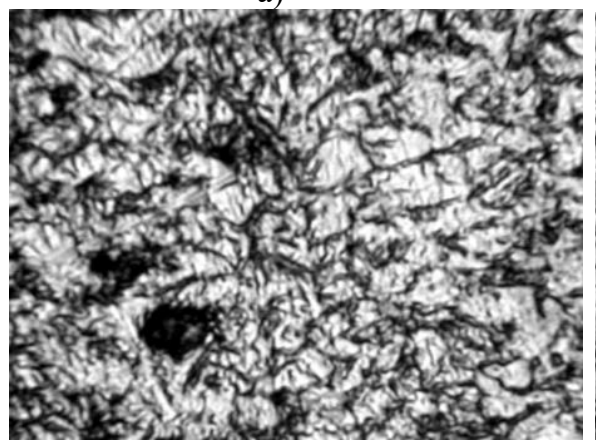

c)

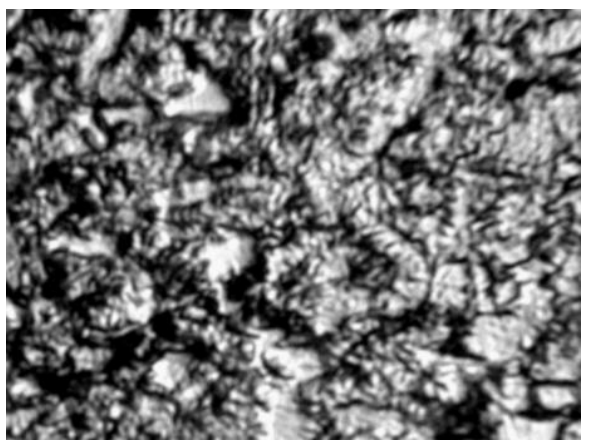

b)

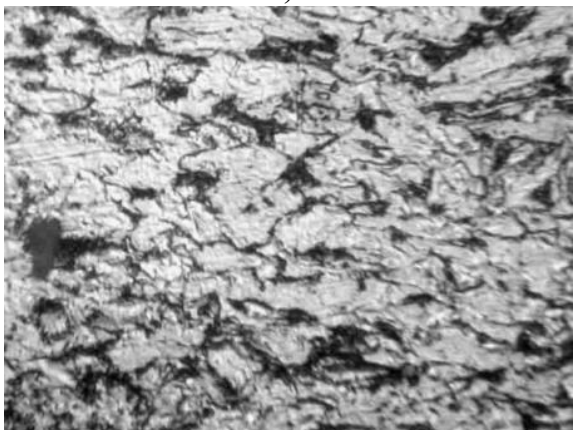

d)

Fig. 5. The microstructure of steel 10HSND, x650: a) - state of factory supply, b) - d) - after deformation by the degree $\varepsilon=15,30,50 \%$.

Studies have shown that in structural steels 08PS, 09G2S and 10HSND there is a clear relationship between the degree of cold plastic deformation, the magnetic parameter Hp and structural changes in structural steels, which makes it possible to recommend a passive flux-probe method for monitoring structural changes in a metal undergoing cold plastic deformation.

\section{Conclusion}

1. The analysis is carried out and the issues of the influence of various factors on the damageability of welded metal structures during long-term operation are examined.

2. A relationship has been established between the scattering magnetic field strength $\mathrm{Hp}$ and the microstructure change in low-carbon steel 08PS and low-alloy steels 09G2S and 10HSND during fractional rolling to different degrees of deformation.

3. It was shown that a significant change in the magnetic field intensity of scattering Hp is characteristic of small degrees of deformation. With a subsequent increase in the degree of deformation, a significant drop in the magnetic parameter $\mathrm{Hp}$ is observed to values approaching negative values of the Earth's magnetic field.

4. The data of microstructural analysis of structural steels are in good agreement with the data of the passive flux-gate control method.

5. A methodology has been developed for assessing the effect of cold plastic deformation on structural changes in metal in welded joints and elements of welded metal structures, which can improve the reliability of assessing the state of the metal in hazardous local zones of SC, especially where it is possible to pass strain to one degree or another, using passive fluxgate control method. 


\section{References}

1. V.E. Gordienko, Technical diagnosis of building structures. Quality control methods: monograph, 144, SPbGASU, SPb (2004)

2. V.E. Gordienko, Control. Diagnostics, No. 3, 45-47 (2005)

3. V.E. Gordienko, E.G. Gordienko, et al., Bulletin of civil engineers, No. 4 (21), 88-90 (2009)

4. V.E. Gordienko, E.G. Gordienko, et al., Bulletin of civil engineers, No. 3 (28), 92-95 (2011)

5. V.E. Gordienko, E.G. Gordienko, et al., Bulletin of civil engineers, No. 4 (29), 114 117 (2011)

6. P. Chaudhari, Science and technology, 42-44, 46-49, 81 (1968)

7. V.E. Gordienko, A.A. Abrosimova, et al., Bulletin of civil engineers, No. 6 (65), 233238 (2017)

8. V.E. Gordienko, Magnetic control and assessment of the stress-strain state of a metal under elastic-plastic deformation: monograph, 144, SPbGASU, SPb (2008)

9. V.E. Gordienko, Magnetic control of structural changes in structural steels under deformation and thermal stresses: monograph, 92, SPbGASU, SPb (2008)

10. V.E. Gordienko, Control. Diagnostics, No. 5, 70-71 (2008)

11. V.E. Gordienko, Bulletin of civil engineers, No. 1 (22), 37-42 (2010)

12. V.E. Gordienko, Passive flux-gate control of the metal structure and internal stresses in the elements of welded MK: monograph, 110, SPbGASU, SPb (2010)

13. V.E. Gordienko, E.G. Gordienko, et al., Industrial and civil engineering, No. 11, 81-83 (2013)

14. V.E. Gordienko, E.V. Trunova, et al., Bulletin of civil engineers, No. 3 (56), 193-197 (2006)

15. V.E. Gordienko, A. A. Abrosimova, Passive flux-gate control and calculation of welded metal structures of building machines taking into account the kinetics of corrosion transformations: monograph, 170, SPbGASU, SPb (2018)

16. V.E. Gordienko, Industrial and civil engineering, No. 12, 42-43 (2005)

17. V.E. Gordienko, N.V. Ovchinnikov, Control. Diagnostics, No. 9, 27-29 (2008)

18. V.E. Gordienko, Bulletin of Civil Engineers, No. 2 (31), 206-209 (2012)

19. A.I. Smirnov, The effect of macro-, meso- and microdefects of the structure on the structural strength of carbon steels under cyclic loading [Text]: author. diss. cand. tech. sciences, 18, Novosibirsk (2003)

20. A.A. Dubov, A.A. Dubov, S.M. Kolokolnikov, Method of magnetic memory (IMF) of metal and control devices (Tissot, Moscow, 2003) 